\title{
New Results of Global Exponential Stabilization for BLDCMs System
}

\author{
Fengxia Tian, ${ }^{1}$ Fangchao Zhen, ${ }^{1}$ Guopeng Zhou, ${ }^{1}$ and Xiaoxin Liao ${ }^{1,2}$ \\ ${ }^{1}$ Institute of Engineering and Technology, Hubei University of Science and Technology, Xianning 437100, China \\ ${ }^{2}$ College of Automation, Huazhong University of Science and Technology, Wuhan 430074, China \\ Correspondence should be addressed to Fangchao Zhen; 2598778594@qq.com
}

Received 16 March 2015; Accepted 27 May 2015

Academic Editor: Zenghui Wang

Copyright (c) 2015 Fengxia Tian et al. This is an open access article distributed under the Creative Commons Attribution License, which permits unrestricted use, distribution, and reproduction in any medium, provided the original work is properly cited.

\begin{abstract}
The global exponential stabilization for brushless direct current motor (BLDCM) system is studied. Four linear and simple feedback controllers are proposed to realize the global stabilization of BLDCM with exponential convergence rate; the control law used in each theorem is less conservative and more concise. Finally, an example is given to demonstrate the correctness of the proposed results.
\end{abstract}

\section{Introduction}

A brushless direct current motor (BLDCM) is a synchronous electric motor which is powered by direct current electricity [1]. With the development of electronic technology and control technology, the BLDCM is used widely in motion control applications. The major advantage of BLDCM is the elimination of the physical contact between the mechanical brushes and the commutators [2]. However, it is found that, with certain values of system parameters, the BLDCM exhibits chaotic behavior, such as aperiodic, random, sudden, or intermittent morbid oscillations of the motor angle speed, which can seriously destroy the stable operation of the motor and can even induce industrial drive system collapse [3-8]. Therefore, studying the stability of BLDCM is a significant job and has been receiving considerable attention.

There has been a lot of work done in studying stability and designing controller of BLDCMs [1, 2, 9-13]. For instance, Dadras et al. studied the stability of chaotic uncertain BLDCMs by feedback linearization and slidingmode control methods [1]. Ge and Chang studied the chaos synchronization and parameters identification of single timescale BLDCMs by adaptive control and backstepping design method [2]. Ren and Chen proposed a piecewise quadratic state feedback method to control chaos in the BLDCM [9]. Meng et al. designed a fuzzy controller with time-delay feedback to control the chaos movement in the BLDCM [10].
Uyaroğlu and Cevher studied sliding-mode control for single time-scale BLDCM [13]. But the controllers proposed in these papers are nonlinear or multiple, and the stability is uniform or asymptotic, which is a weaker property than exponential stability. So, searching a lower-dimensional linear control for the global exponential stabilization of chaotic BLDCM is significant.

Recently, Wei et al. studied the global exponential stabilization for chaotic BLDCMs based on Lyapunov-like theorem [14], although Wei et al. present the method to address the problem about global exponential stability of the BLDCM system firstly. It is innovative to generalize stability and asymptotic stability to global exponential stability and generalize nonlinear feedback control law to linear feedback control law which is easier to implement. There is only one theorem and the control law is not concise. In this paper, we further improved the results of Wei et al. and gave four theorems. The control law used in each theorem is less conservative and more concise, and the proofs are much more simple.

The rest of this paper is organized as follows. In Section 2, system model is presented. In Section 3, the main results of global exponential stabilization for BLDCM system are derived. In Section 4, one example is presented to demonstrate the effectivity of the proposed method. The final section is the conclusion. 


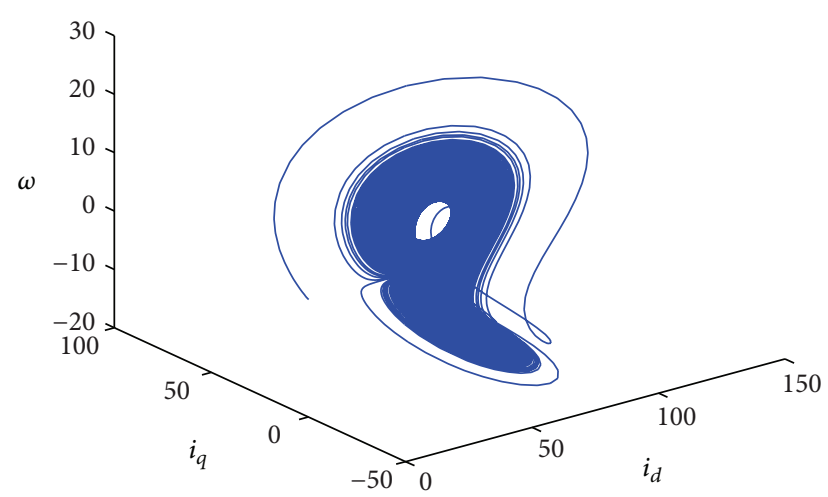

FIGURE 1: The typical chaotic attractor of the BLDCM at $\sigma=4, \gamma=$ 55 , and $\delta=0.875$.

\section{Preliminaries}

Consider the mathematical model of a BLDC motor as follows [3-7]:

$$
\begin{aligned}
& \frac{\mathrm{d} i_{d}}{\mathrm{~d} t}=U_{d}-\delta i_{d}+i_{q} \omega \\
& \frac{\mathrm{d} i_{q}}{\mathrm{~d} t}=U_{q}-i_{q}-i_{d} \omega+\gamma \omega \\
& \frac{\mathrm{d} \omega}{\mathrm{d} t}=\sigma i_{q}-\sigma \omega-T_{L},
\end{aligned}
$$

where $i_{d}, i_{q}$, and $\omega$ denote quadrature-axis current, direct-axis current, and angle speed, respectively. $\delta, \gamma$, and $\sigma$ are system parameters, which determine the type of the dynamical regime of the motor. Consider the case that the motor is running freely under no loading conditions; that is, $U_{d}=$ $U_{q}=T_{L}=0$, so system (1) becomes

$$
\begin{aligned}
& \frac{\mathrm{d} i_{d}}{\mathrm{~d} t}=-\delta i_{d}+i_{q} \omega \\
& \frac{\mathrm{d} i_{q}}{\mathrm{~d} t}=-i_{q}-i_{d} \omega+\gamma \omega \\
& \frac{\mathrm{d} \omega}{\mathrm{d} t}=\sigma i_{q}-\sigma \omega .
\end{aligned}
$$

System (2) has chaotic behaviors, with a chaotic attractor shown in Figure 1.

From Figure 1 one can see that the BLDCM is not stable and shows some chaos characteristics, which led to the unstable operation of the motor which is not acceptable in real applications. Therefore, studying stabilization for chaotic BLDCM is significant and this paper will work on it.

Now, some necessary definitions [15-18] that will be used in this paper are introduced in the following. Consider a general nonautonomous system of differential equation:

$$
\frac{\mathrm{d} x}{\mathrm{~d} t}=f(x, t),
$$

where $x=\left[x_{1}, x_{2}, \ldots, x_{n}\right]^{\mathrm{T}}$ is the state vector, $f=\left[f_{1}, f_{2}, \ldots\right.$, $\left.f_{n}\right]^{\mathrm{T}} \in R^{n \times 1}$ are continuous functions with respect to $x$, and $f(0)=0$. Furthermore, $V(x)$ is a radially unbounded and positive definite Lyapunov function.

Definition 1. The trivial solution of system (3) is globally exponentially stable if, for any $x_{0} \in R^{n}$, there exist two constants $M\left(x_{0}\right)>0$ and $\alpha>0$, such that, for any solution of system (3), it holds

$$
\|x(t)\| \leq M\left(x_{0}\right) e^{-\alpha\left(t-t_{0}\right)},
$$

where $x(t)=x\left(t, t_{0}, x_{0}\right)$.

Lemma 2. If there exists a Lyapunov function $V(t, x)$ satisfying

$$
\begin{aligned}
\|x(t)\| & \leq V(t, x), \\
\left.\frac{\mathrm{d} V(x)}{\mathrm{d} t}\right|_{(3)} & \leq-c V(x),
\end{aligned}
$$

where $c>$ constant $>0$, then the trivial solution of system (3) is globally exponentially stable.

$$
\begin{aligned}
\text { Proof. } \because V(x(t)) & \leq V\left(x\left(t_{0}\right)\right) e^{-c\left(t-t_{0}\right)} \text {, then } \\
\|x(t)\| & \leq\|V(x(t))\| \leq\left\|V\left(x\left(t_{0}\right)\right) e^{-c\left(t-t_{0}\right)}\right\| \\
& =M\left(x_{0}\right) e^{-c\left(t-t_{0}\right)} .
\end{aligned}
$$

So the trivial solution of system (3) is globally exponentially stable.

\section{Main Results}

Theorem 3. Add a linear feedback controller $u_{1}=-k_{2} i_{q}$ to the second equation of system (2), and then the system can be described as

$$
\begin{aligned}
& \frac{\mathrm{d} i_{d}}{\mathrm{~d} t}=-\delta i_{d}+i_{q} \omega \\
& \frac{\mathrm{d} i_{q}}{\mathrm{~d} t}=-\left(1+k_{2}\right) i_{q}-i_{d} \omega+\gamma \omega \\
& \frac{\mathrm{d} \omega}{\mathrm{d} t}=\sigma i_{q}-\sigma \omega,
\end{aligned}
$$

or add a linear feedback controller $u_{2}=-k_{3} \omega$ to the third equation of system (2), and then the system can be described as

$$
\begin{aligned}
& \frac{\mathrm{d} i_{d}}{\mathrm{~d} t}=-\delta i_{d}+i_{q} \omega \\
& \frac{\mathrm{d} i_{q}}{\mathrm{~d} t}=-i_{q}-i_{d} \omega+\gamma \omega \\
& \frac{\mathrm{d} \omega}{\mathrm{d} t}=\sigma i_{q}-\left(\sigma+k_{3}\right) \omega .
\end{aligned}
$$

If $\left(\begin{array}{cc}-1-k_{2} & (\gamma+\sigma) / 2 \\ (\gamma+\sigma) / 2 & -\sigma\end{array}\right)$ is negative definite, which means

$$
k_{2}>\frac{(\gamma+\sigma)^{2}}{4 \sigma}-1
$$


then system (7) is globally exponentially stable at the equilibrium point $\left(i_{d}, i_{q}, \omega\right)=(0,0,0)$.

And if $\left(\begin{array}{cc}-1 & (\gamma+\sigma) / 2 \\ (\gamma+\sigma) / 2 & -\sigma-k_{3}\end{array}\right)$ is negative definite, which means

$$
k_{3}>\left(\frac{\gamma+\sigma}{2}\right)^{2}-\sigma
$$

then system (8) is globally exponentially stable at the equilibrium point $\left(i_{d}, i_{q}, \omega\right)=(0,0,0)$.

Proof. Construct a radially unbounded and positive definite Lyapunov function:

$$
V_{1}=\frac{1}{2}\left[\left(i_{d}\right)^{2}+\left(i_{q}\right)^{2}+\omega^{2}\right]
$$

(1) Because $k_{2}>(\gamma+\sigma)^{2} / 4 \sigma-1,\left(\begin{array}{cc}-1-k_{2} & (\gamma+\sigma) / 2 \\ (\gamma+\sigma) / 2 & -\sigma\end{array}\right)$ is negative definite. So

$$
\phi_{1}=\left(\begin{array}{ccc}
-\delta & 0 & 0 \\
0 & -1-k_{2} & \frac{\gamma+\sigma}{2} \\
0 & \frac{\gamma+\sigma}{2} & -\sigma
\end{array}\right)
$$

is negative definite.

Compute the derivative of $V_{1}$ along system (7):

$$
\begin{aligned}
& \left.\frac{\mathrm{d} V_{1}}{\mathrm{~d} t}\right|_{(7)}=i_{d} \dot{i}_{d}+i_{q} \dot{i}_{q}+\omega \dot{\omega} \\
& =-\delta i_{d}^{2}+i_{d} i_{q} \omega-\left(1+k_{2}\right) i_{q}^{2}-i_{d} i_{q} \omega+\gamma \omega i_{q} \\
& +\sigma i_{q} \omega-\sigma \omega^{2} \\
& =-\delta i_{d}^{2}-\left(1+k_{2}\right) i_{q}^{2}-\sigma \omega^{2}+(\gamma+\sigma) \omega i_{q} \\
& =\left(\begin{array}{c}
i_{d} \\
i_{q} \\
\omega
\end{array}\right)^{T}\left(\begin{array}{ccc}
-\delta & 0 & 0 \\
0 & -1-k_{2} & \frac{\gamma+\sigma}{2} \\
0 & \frac{\gamma+\sigma}{2} & -\sigma
\end{array}\right)\left(\begin{array}{c}
i_{d} \\
i_{q} \\
\omega
\end{array}\right) \\
& =\left(\begin{array}{c}
i_{d} \\
i_{q} \\
\omega
\end{array}\right)^{T} \phi_{1}\left(\begin{array}{c}
i_{d} \\
i_{q} \\
\omega
\end{array}\right) \\
& \leq \lambda_{M}\left(\phi_{1}\right)\left(\begin{array}{c}
i_{d} \\
i_{q} \\
\omega
\end{array}\right)^{T}\left(\begin{array}{c}
i_{d} \\
i_{q} \\
\omega
\end{array}\right) \leq 2 \lambda_{M}\left(\phi_{1}\right) V_{1} \text {, }
\end{aligned}
$$

where $\lambda_{M}\left(\phi_{1}\right)$ is the largest eigenvalue of $\phi_{1}$ and $\lambda_{M}\left(\phi_{1}\right)<0$. Consider

$$
\begin{aligned}
V_{1}(t) & \leq V_{1}\left(t_{0}\right) e^{2 \lambda_{M}\left(\phi_{1}\right)\left(t-t_{0}\right)} \\
i_{d}^{2}(t)+i_{q}^{2}(t)+\omega^{2}(t) & \leq 2 V_{1}\left(t_{0}\right) e^{2 \lambda_{M}\left(\phi_{1}\right)\left(t-t_{0}\right)} .
\end{aligned}
$$

So the zero solution of system (7) is globally exponentially stable.

(2) Because $k_{3}>((\gamma+\sigma) / 2)^{2}-\sigma,\left(\begin{array}{cc}-1 & (\gamma+\sigma) / 2 \\ (\gamma+\sigma) / 2 & -\sigma-k_{3}\end{array}\right)$ is negative definite. So

$$
\phi_{2}=\left(\begin{array}{ccc}
-\delta & 0 & 0 \\
0 & -1 & \frac{\gamma+\sigma}{2} \\
0 & \frac{\gamma+\sigma}{2} & -\sigma-k_{3}
\end{array}\right)
$$

is negative definite.

Similarly, compute the derivative of $V_{1}$ along system (8):

$$
\begin{aligned}
\left.\frac{\mathrm{d} V_{1}}{\mathrm{~d} t}\right|_{(8)}= & i_{d} \dot{i}_{d}+i_{q} \dot{i}_{q}+\omega \dot{\omega} \\
= & -\delta i_{d}^{2}+i_{d} i_{q} \omega-i_{q}^{2}-i_{d} i_{q} \omega+\gamma \omega i_{q}+\sigma i_{q} \omega \\
& -\left(\sigma+k_{3}\right) \omega^{2} \\
= & -\delta i_{d}^{2}-i_{q}^{2}-\left(\sigma+k_{3}\right) \omega^{2}+(\gamma+\sigma) \omega i_{q} \\
= & \left(\begin{array}{c}
i_{d} \\
i_{q} \\
\omega
\end{array}\right)^{T}\left(\begin{array}{cc}
-\delta & 0 \\
0 & -1 \\
0 & \frac{\gamma+\sigma}{2}
\end{array}\right)\left(\begin{array}{c}
i_{d} \\
i_{q} \\
\omega
\end{array}\right) \\
= & \left(\begin{array}{c}
i_{d} \\
i_{q} \\
\omega
\end{array}\right)^{T}\left(\begin{array}{c}
i_{d} \\
i_{q} \\
\phi_{2}
\end{array}\right) \\
\leq & \lambda_{M}\left(\phi_{2}\right)\left(\begin{array}{l}
i_{d} \\
i_{q} \\
\omega
\end{array}\right)\left(\begin{array}{l}
i_{d} \\
i_{q} \\
\omega
\end{array}\right) \leq 2 \lambda_{M}\left(\phi_{2}\right) V_{1},
\end{aligned}
$$

where $\lambda_{M}\left(\phi_{2}\right)$ is the largest eigenvector of $\phi_{2}$ and $\lambda_{M}\left(\phi_{2}\right)<0$. Consider

$$
\begin{aligned}
V_{1}(t) & \leq V_{1}\left(t_{0}\right) e^{2 \lambda_{M}\left(\phi_{2}\right)\left(t-t_{0}\right)} \\
i_{d}^{2}(t)+i_{q}^{2}(t)+\omega^{2}(t) & \leq 2 V_{1}\left(t_{0}\right) e^{2 \lambda_{M}\left(\phi_{2}\right)\left(t-t_{0}\right)} .
\end{aligned}
$$

So the zero solution of system (8) is globally exponentially stable.

Therefore, systems (7) and (8) are globally exponentially stable at the equilibrium point $s_{0}=(0,0,0)$.

Theorem 4. Add a linear negative feedback controller $u_{3}=$ $-\tilde{\gamma} \omega$ to the second equation of system (2), and then the system can be described as

$$
\begin{aligned}
& \frac{\mathrm{d} i_{d}}{\mathrm{~d} t}=-\delta i_{d}+i_{q} \omega \\
& \frac{\mathrm{d} i_{q}}{\mathrm{~d} t}=-i_{q}-i_{d} \omega+(\gamma-\widetilde{\gamma}) \omega \\
& \frac{\mathrm{d} \omega}{\mathrm{d} t}=\sigma i_{q}-\sigma \omega ;
\end{aligned}
$$


if $\tilde{\gamma} \geq \gamma$, system (18) is globally exponentially stable at the equilibrium point $\left(i_{d}, i_{q}, \omega\right)=(0,0,0)$.

Proof. (1) If $\tilde{\gamma}>\gamma$, construct a radially unbounded and positive definite Lyapunov function:

$$
\begin{aligned}
V_{2} & =\frac{1}{2}\left[\left(i_{d}\right)^{2}+\left(i_{q}\right)^{2}+\frac{\tilde{\gamma}-\gamma}{\sigma} \omega^{2}\right], \\
\min & {\left[1, \frac{\tilde{\gamma}-\gamma}{\sigma}\right]\left(i_{d}^{2}+i_{q}^{2}+\omega^{2}\right) \leq 2 V_{2} } \\
& \leq \max \left[1, \frac{\tilde{\gamma}-\gamma}{\sigma}\right]\left(i_{d}^{2}+i_{q}^{2}+\omega^{2}\right) .
\end{aligned}
$$

Compute the derivative of $V_{2}$ along system (18):

$$
\begin{aligned}
& \left.\frac{\mathrm{d} V_{2}}{\mathrm{~d} t}\right|_{(18)}=i_{d} \dot{i}_{d}+i_{q} \dot{i}_{q}+\frac{\tilde{\gamma}-\gamma}{\sigma} \omega \dot{\omega}=-\delta i_{d}^{2}+i_{d} i_{q} \omega-i_{q}^{2} \\
& -i_{d} i_{q} \omega+(\gamma-\tilde{\gamma}) \omega i_{q}+\frac{\tilde{\gamma}-\gamma}{\sigma} \sigma i_{q} \omega-\frac{\tilde{\gamma}-\gamma}{\sigma} \sigma \omega^{2} \\
& =-\delta i_{d}^{2}-i_{q}^{2}-(\tilde{\gamma}-\gamma) \omega^{2}=\left(\begin{array}{c}
i_{d} \\
i_{q} \\
\omega
\end{array}\right)^{T} \\
& \cdot\left(\begin{array}{ccc}
-\delta & 0 & 0 \\
0 & -1 & 0 \\
0 & 0 & \tilde{\gamma}-\gamma
\end{array}\right)\left(\begin{array}{c}
i_{d} \\
i_{q} \\
\omega
\end{array}\right) \\
& \leq-\min [\sigma, 1, \tilde{\gamma}-\gamma]\left(i_{d}^{2}+i_{q}^{2}+\omega^{2}\right) \\
& \leq-\frac{\min [\sigma, 1, \tilde{\gamma}-\gamma]}{\max [1,(\tilde{\gamma}-\gamma) / \sigma]} 2 V_{2} \\
& V_{2} \leq V_{2}\left(t_{0}\right) e^{-(2 \min [\sigma, 1, \tilde{\gamma}-\gamma] / \max [1,(\tilde{\gamma}-\gamma) / \sigma])\left(t-t_{0}\right)} \\
& i_{d}^{2}(t)+i_{q}^{2}(t)+\omega^{2}(t) \leq \frac{2 V_{2}\left(t_{0}\right)}{\min [1,(\tilde{\gamma}-\gamma) / \sigma]} \\
& \cdot e^{-(2 \min [\sigma, 1, \tilde{\gamma}-\gamma] / \max [1,(\tilde{\gamma}-\gamma) / \sigma])\left(t-t_{0}\right)} .
\end{aligned}
$$

So the zero solution of system (18) is globally exponentially stable.

(2) If $\tilde{\gamma}=\gamma$, system (18) changes as

$$
\begin{aligned}
& \frac{\mathrm{d} i_{d}}{\mathrm{~d} t}=-\delta i_{d}+i_{q} \omega \\
& \frac{\mathrm{d} i_{q}}{\mathrm{~d} t}=-i_{q}-i_{d} \omega \\
& \frac{\mathrm{d} \omega}{\mathrm{d} t}=\sigma i_{q}-\sigma \omega .
\end{aligned}
$$

For the first two equations, construct a radially unbounded and positive definite Lyapunov function:

$$
V_{3}=\frac{1}{2}\left[\left(i_{d}\right)^{2}+\left(i_{q}\right)^{2}\right]
$$

Compute the derivative of $V_{3}$ along system (21):

$$
\begin{aligned}
\left.\frac{\mathrm{d} V_{3}}{\mathrm{~d} t}\right|_{(21)} & =i_{d} \dot{i}_{d}+i_{q} i_{q}=-\delta i_{d}^{2}+i_{d} i_{q} \omega-i_{q}^{2}-i_{d} i_{q} \omega \\
& =-\delta i_{d}^{2}-i_{q}^{2} \leq-\min [\delta, 1]\left(i_{d}^{2}+i_{q}^{2}\right) \\
& =-\min [\delta, 1] 2 V_{3} \\
V_{3} & \leq V_{3}\left(t_{0}\right) e^{-\min [\delta, 1]\left(t-t_{0}\right)} \\
i_{d}^{2}(t)+i_{q}^{2}(t) & \leq 2 V_{3}\left(t_{0}\right) e^{-\min [\delta, 1]\left(t-t_{0}\right)} .
\end{aligned}
$$

So the zero solution of system (21) is globally exponentially stable on partial variables $i_{q}, i_{d}$.

For the third equation of system (21)

$$
\begin{aligned}
\omega( & t)=\omega\left(t_{0}\right) e^{-\sigma\left(t-t_{0}\right)}+\int_{t_{0}}^{t} e^{-\sigma(t-\tau)} \sigma i_{q}(\tau) d \tau \\
\leq & \left|\omega\left(t_{0}\right)\right| e^{-\sigma\left(t-t_{0}\right)}+\int_{t_{0}}^{t} e^{-\sigma(t-\tau)} \sigma\left|i_{q}(\tau)\right| d \tau \\
\leq & \left|\omega\left(t_{0}\right)\right| e^{-\sigma\left(t-t_{0}\right)} \\
+ & \sigma e^{-\sigma t} \int_{t_{0}}^{t} e^{\sigma \tau} \sqrt{\left(i_{q}(\tau)\right)^{2}+\left(i_{d}(\tau)\right)^{2}} d \tau \\
\leq & \left|\omega\left(t_{0}\right)\right| e^{-\sigma\left(t-t_{0}\right)} \\
+ & \sigma e^{-\sigma t} \int_{t_{0}}^{t} e^{\sigma \tau} \sqrt{\left(i_{q}\left(t_{0}\right)\right)^{2}+\left(i_{d}\left(t_{0}\right)\right)^{2}} e^{-\min (\delta, 1)\left(\tau-t_{0}\right)} d \tau \\
\leq & \left|\omega\left(t_{0}\right)\right| e^{-\sigma\left(t-t_{0}\right)}+\sigma e^{-\sigma t} \int_{t_{0}}^{t} e^{\sigma \tau} H e^{-\alpha\left(\tau-t_{0}\right)} d \tau \\
\leq & \leq\left|\omega\left(t_{0}\right)\right| e^{-\sigma\left(t-t_{0}\right)}+\sigma H e^{-\sigma t} e^{\alpha t_{0}} \int_{t_{0}}^{t} e^{(\sigma-\alpha) \tau} d \tau \\
\leq & \left.\leq\left|\omega\left(t_{0}\right)\right|-\frac{\sigma H}{\sigma-\alpha}\right) e^{-\sigma\left(t-t_{0}\right)}+\frac{\sigma H}{\sigma-\alpha} e^{-\alpha\left(t-t_{0}\right)}, \\
\leq & \left.\mid t_{0}\right) \mid e^{-\sigma\left(t-t_{0}\right)}+\sigma H e^{-\sigma t} e^{\alpha t_{0}} \frac{e^{(\sigma-\alpha) t}-e^{(\sigma-\alpha) t_{0}}}{\sigma-\alpha} \\
&
\end{aligned}
$$

where we can suppose that $H=\sqrt{\left(i_{q}\left(t_{0}\right)\right)^{2}+\left(i_{d}\left(t_{0}\right)\right)^{2}}, \alpha=$ $\min (\delta, 1)$.

So the zero solution of system (21) is globally exponentially stable on partial variables $\omega$.

Therefore system (18) is globally exponentially stable on all variables $\left(i_{d}, i_{q}, \omega\right)$ at the equilibrium point $\left(i_{d}, i_{q}, \omega\right)=$ $(0,0,0)$. 
Theorem 5. Add a linear negative feedback controller $u_{4}=$ $-\widetilde{\sigma} i_{q}$ to the third equation of system (2), and then the system can be described as

$$
\begin{aligned}
& \frac{\mathrm{d} i_{d}}{\mathrm{~d} t}=-\delta i_{d}+i_{q} \omega \\
& \frac{\mathrm{d} i_{q}}{\mathrm{~d} t}=-i_{q}-i_{d} \omega+\gamma \omega \\
& \frac{\mathrm{d} \omega}{\mathrm{d} t}=(\sigma-\widetilde{\sigma}) i_{q}-\sigma \omega ;
\end{aligned}
$$

if $\widetilde{\sigma} \geq \sigma$, system (25) is globally exponentially stable at the equilibrium point $\left(i_{d}, i_{q}, \omega\right)=(0,0,0)$.

Proof. (1) If $\widetilde{\sigma}>\sigma$, construct a radially unbounded and positive definite Lyapunov function:

$$
\begin{aligned}
V_{4} & =\frac{1}{2}\left[\left(i_{d}\right)^{2}+\left(i_{q}\right)^{2}+\frac{\gamma}{\widetilde{\sigma}-\sigma} \omega^{2}\right], \\
\min & {\left[1, \frac{\gamma}{\widetilde{\sigma}-\sigma}\right]\left(i_{d}^{2}+i_{q}^{2}+\omega^{2}\right) \leq 2 V_{4} } \\
& \leq \max \left[1, \frac{\gamma}{\widetilde{\sigma}-\sigma}\right]\left(i_{d}^{2}+i_{q}^{2}+\omega^{2}\right) .
\end{aligned}
$$

Compute the derivative of $V_{4}$ along system (25):

$$
\begin{aligned}
& \left.\frac{\mathrm{d} V_{4}}{\mathrm{~d} t}\right|_{(25)}=i_{d} i_{d}+i_{q} i_{q}+\frac{\gamma}{\widetilde{\sigma}-\sigma} \omega \dot{\omega}=-\delta i_{d}^{2}+i_{d} i_{q} \omega-i_{q}^{2} \\
& -i_{d} i_{q} \omega+\gamma \omega i_{q}+\frac{\gamma}{\widetilde{\sigma}-\sigma}(\sigma-\widetilde{\sigma}) i_{q} \omega-\frac{\gamma}{\widetilde{\sigma}-\sigma} \sigma \omega^{2} \\
& \quad=-\delta i_{d}^{2}-i_{q}^{2}-\frac{\gamma \sigma}{\widetilde{\sigma}-\sigma} \omega^{2} \\
& \leq-\min [\delta, 1, \gamma \sigma /(\widetilde{\sigma}-\sigma)]\left(i_{d}^{2}+i_{q}^{2}+\omega^{2}\right) \\
& \leq-\frac{\min [\sigma, 1, \gamma \sigma /(\widetilde{\sigma}-\sigma)]}{\max [1, \gamma /(\widetilde{\sigma}-\sigma)]} 2 V_{4} \\
& V_{4}(t) \leq V_{4}\left(t_{0}\right) e^{-(2 \min [\sigma, \gamma \sigma /(\widetilde{\sigma}-\sigma)] / \max [1, \gamma /(\widetilde{\sigma}-\sigma)])\left(t-t_{0}\right)} \\
& i_{d}^{2}(t)+i_{q}^{2}(t)+\omega^{2}(t) \leq \frac{2 V_{4}\left(t_{0}\right)}{\min [1, \gamma /(\widetilde{\sigma}-\sigma)]} \\
& \cdot e^{-(2 \min [\sigma, \gamma \sigma /(\widetilde{\sigma}-\sigma)] / \max [1, \gamma /(\widetilde{\sigma}-\sigma)])\left(t-t_{0}\right)} .
\end{aligned}
$$

When $\widetilde{\sigma}>\sigma$, the zero solution of system (25) is globally exponentially stable.

(2) If $\widetilde{\sigma}=\sigma$, the system is

$$
\begin{aligned}
& \frac{\mathrm{d} i_{d}}{\mathrm{~d} t}=-\delta i_{d}+i_{q} \omega \\
& \frac{\mathrm{d} i_{q}}{\mathrm{~d} t}=-i_{q}-i_{d} \omega+\gamma \omega \\
& \frac{\mathrm{d} \omega}{\mathrm{d} t}=-\sigma \omega .
\end{aligned}
$$

Because $\left(\begin{array}{cc}-1 & \gamma / 2 \\ \gamma / 2 & 0\end{array}\right)$ is negative definite,

$$
\left(\begin{array}{ccc}
-\delta & 0 & 0 \\
0 & -1 & \frac{\gamma}{2} \\
0 & \frac{\gamma}{2} & 0
\end{array}\right)
$$

is negative definite.

For the first two equations, compute the derivative of $V_{3}$ along system (28):

$$
\begin{aligned}
\frac{\left.\mathrm{d} V_{3}\right|_{(28)}}{\mathrm{d} t} & =i_{d} \dot{i}_{d}+i_{q} i_{q} \\
& =-\delta i_{d}^{2}+i_{d} i_{q} \omega-i_{q}^{2}-i_{d} i_{q} \omega+\gamma \omega i_{q} \\
& =-\delta i_{d}^{2}-i_{q}^{2}+\gamma \omega i_{q} \\
& =\left(\begin{array}{c}
i_{d} \\
i_{q} \\
\omega
\end{array}\right)^{T}\left(\begin{array}{ccc}
-\delta & 0 & 0 \\
0 & -1 & \frac{\gamma}{2} \\
0 & \frac{\gamma}{2} & 0
\end{array}\right)\left(\begin{array}{c}
i_{d} \\
i_{q} \\
\omega
\end{array}\right) \\
& \left.=\left(\begin{array}{c}
i_{d} \\
i_{q} \\
\omega
\end{array}\right) \phi_{3}^{T}\left(\begin{array}{c}
i_{d} \\
i_{q} \\
\omega
\end{array}\right) \begin{array}{l}
i_{d}^{T} \\
i_{q} \\
\omega
\end{array}\right)\left(\begin{array}{c}
i_{d} \\
i_{q} \\
\omega
\end{array}\right) \leq 2 \lambda_{M}\left(\phi_{3}\right) V_{3}, \\
& \leq \lambda_{M}\left(\phi_{3}\right)
\end{aligned}
$$

where $\lambda_{M}\left(\phi_{3}\right)$ is the largest eigenvector of $\phi_{3}$ and $\lambda_{M}\left(\phi_{3}\right)<0$. Consider

$$
\begin{aligned}
V_{3} & \leq V_{3}\left(t_{0}\right) e^{\lambda_{M}\left(\phi_{3}\right)\left(t-t_{0}\right)} \\
i_{d}^{2}(t)+i_{q}^{2}(t) & \leq 2 V_{3}\left(t_{0}\right) e^{\lambda_{M}\left(\phi_{3}\right)\left(t-t_{0}\right)} .
\end{aligned}
$$

When $\widetilde{\sigma}=\sigma$, the zero solution of system (25) is globally exponentially stable on partial variables $i_{d}, i_{q}$.

For the third equation of system (28)

$$
\omega(t)=\omega\left(t_{0}\right) e^{-\sigma\left(t-t_{0}\right)}
$$

So the zero solution of system (28) is globally exponentially stable on partial variable $\omega$.

Therefore, system (25) is globally exponentially stable on all variables $i_{d}, i_{q}$, and $\omega$ at the equilibrium point $\left(i_{d}, i_{q}, \omega\right)=$ $(0,0,0)$. 
Theorem 6. Add linear negative feedback controllers $u_{3}=$ $-\tilde{\gamma} \omega, u_{4}=-\widetilde{\sigma} i_{q}$ to the second and third equations of system (2) simultaneously; then the system can be described as

$$
\begin{aligned}
& \frac{\mathrm{d} i_{d}}{\mathrm{~d} t}=-\delta i_{d}+i_{q} \omega \\
& \frac{\mathrm{d} i_{q}}{\mathrm{~d} t}=-i_{q}-i_{d} \omega+(\gamma-\tilde{\gamma}) \omega \\
& \frac{\mathrm{d} \omega}{\mathrm{d} t}=(\sigma-\widetilde{\sigma}) i_{q}-\sigma \omega .
\end{aligned}
$$

If $\tilde{\gamma} \leq \gamma, \widetilde{\sigma} \leq \sigma$ and $0<(\gamma-\tilde{\gamma}) \ll 1,0<(\sigma-\widetilde{\sigma}) \ll 1$, such that following matrix $\left(\begin{array}{cc}-1 & (\gamma-\tilde{\gamma}+\sigma-\widetilde{\sigma}) / 2 \\ (\gamma-\sigma-\widetilde{\sigma}) / 2 & -\sigma\end{array}\right)$ is negative definite. Then system (33) is globally exponentially stable at the equilibrium point $\left(i_{d}, i_{q}, \omega\right)=(0,0,0)$.

Proof. Since $\left(\begin{array}{cc}-1 & (\gamma-\widetilde{\gamma}+\sigma-\widetilde{\sigma}) / 2 \\ (\gamma-\tilde{\gamma}+\sigma-\widetilde{\sigma}) / 2 & -\sigma\end{array}\right)$ is negative definite, $\left(\begin{array}{ccc}-\sigma & 0 & 0 \\ 0 & -1 & (\gamma-\widetilde{\gamma}+\sigma-\widetilde{\sigma}) / 2 \\ 0 & (\gamma-\widetilde{\gamma}+\sigma-\widetilde{\sigma}) / 2 & -\sigma\end{array}\right)$ is negative definite.

Compute the derivative of $V_{1}$ along system (33):

$$
\begin{aligned}
& \left.\frac{\mathrm{d} V_{1}}{\mathrm{~d} t}\right|_{(33)}=i_{d} \dot{i}_{d}+i_{q} \dot{i}_{q}+\omega \dot{\omega}=-\delta i_{d}^{2}+i_{d} i_{q} \omega-i_{q}^{2} \\
& -i_{d} i_{q} \omega+(\gamma-\tilde{\gamma}) \omega i_{q}+(\sigma-\widetilde{\sigma}) i_{q} \omega-\sigma \omega^{2}=-\delta i_{d}^{2} \\
& -i_{q}^{2}-\sigma \omega^{2}+(\gamma-\tilde{\gamma}+\sigma-\tilde{\sigma}) \omega=\left(\begin{array}{c}
i_{d} \\
i_{q} \\
\omega
\end{array}\right)^{T} \\
& \left(\begin{array}{ccc}
-\delta & 0 & 0 \\
0 & -1 & \frac{\gamma-\tilde{\gamma}+\sigma-\tilde{\sigma}}{2} \\
0 & \frac{\gamma-\tilde{\gamma}+\sigma-\tilde{\sigma}}{2} & -\sigma
\end{array}\right)\left(\begin{array}{c}
i_{d} \\
i_{q} \\
\omega
\end{array}\right) \\
& =\left(\begin{array}{c}
i_{d} \\
i_{q} \\
\omega
\end{array}\right)^{T} \phi_{4}\left(\begin{array}{c}
i_{d} \\
i_{q} \\
\omega
\end{array}\right) \leq \lambda_{M}\left(\phi_{4}\right)\left(\begin{array}{c}
i_{d} \\
i_{q} \\
\omega
\end{array}\right)^{T}\left(\begin{array}{c}
i_{d} \\
i_{q} \\
\omega
\end{array}\right) \\
& \leq 2 \lambda_{M}\left(\phi_{4}\right) V \text {, }
\end{aligned}
$$

where $\lambda_{M}\left(\phi_{4}\right)$ is the largest eigenvalue of $\phi_{4}$ and $\lambda_{M}\left(\phi_{4}\right)<0$. Consider

$$
\begin{aligned}
V_{1}(t) & \leq V_{1}\left(t_{0}\right) e^{2 \lambda_{M}\left(\phi_{4}\right)\left(t-t_{0}\right)} \\
i_{d}^{2}(t)+i_{q}^{2}(t)+\omega^{2}(t) & \leq 2 V_{1}\left(t_{0}\right) e^{2 \lambda_{M}\left(\phi_{1}\right)\left(t-t_{0}\right)} .
\end{aligned}
$$

So when $\tilde{\gamma} \geq \gamma, \widetilde{\sigma} \geq \sigma$ and $0<(\gamma-\tilde{\gamma}) \ll 1,0<(\gamma-\widetilde{\gamma}) \ll 1$ system (33) is globally exponentially stable at the equilibrium point $\left(i_{d}, i_{q}, \omega\right)=(0,0,0)$.

\section{Illustrative Example}

The parameters of the motor are selected as $\sigma=4, \gamma=55$, and $\delta=0.875$ and the initial values of the system are chosen as $\left(\omega(0), i_{q}(0), i_{d}(0)\right)=(0.1,0.1,0.1)$.
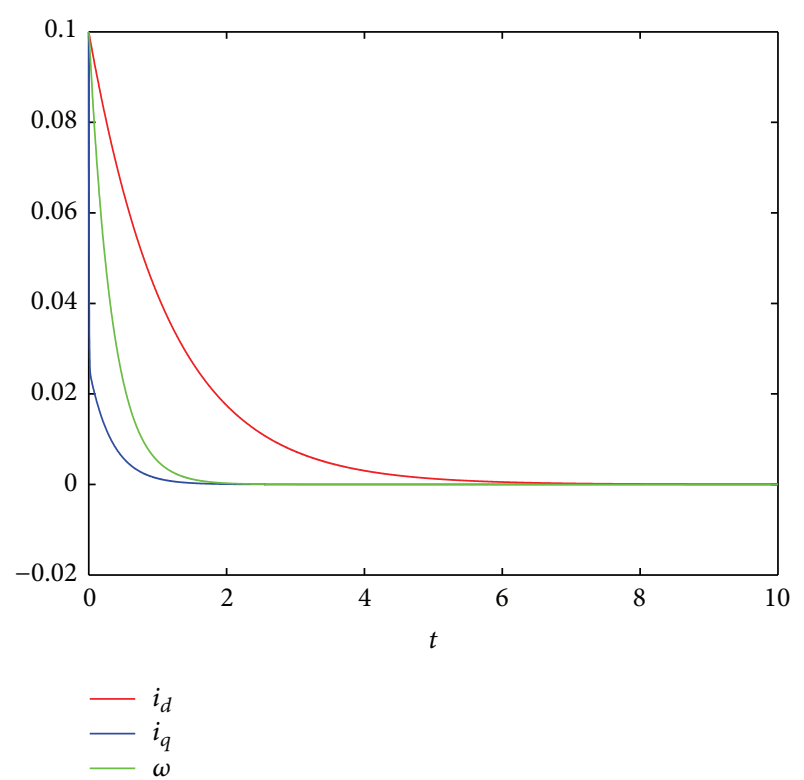

FIgURE 2: The state trajectory of $i_{d}, i_{q}$, and $\omega$ with the controller $u_{1}$.

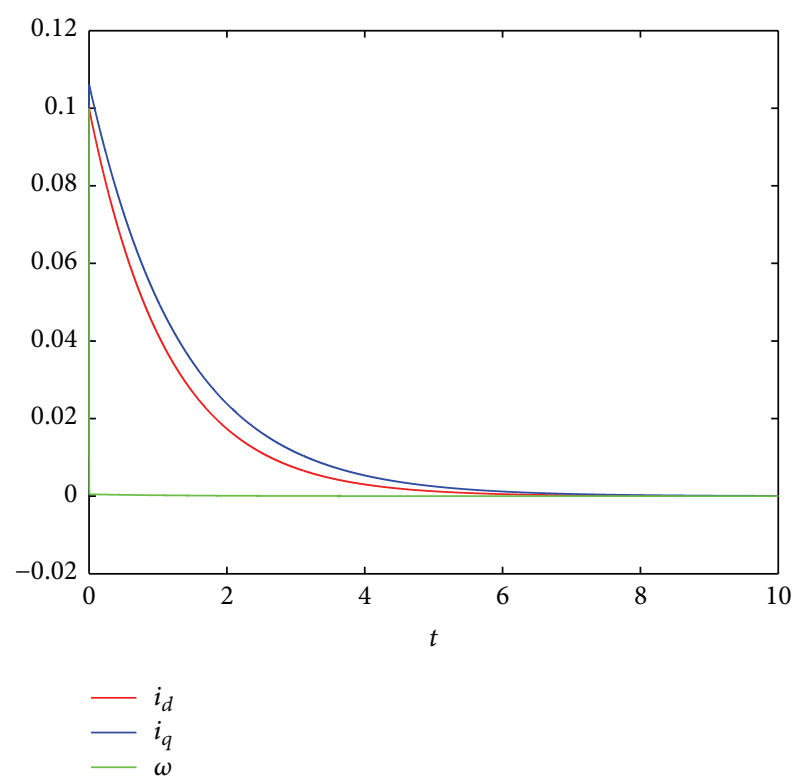

FIgURE 3: The state trajectory of $i_{d}, i_{q}$, and $\omega$ with the controller $u_{2}$.

According to Theorem 3, we may choose $k_{2}=216.6>$ $(\gamma+\sigma)^{2} / 4 \sigma-1=216.5625, k_{3}=867>((\gamma+\sigma) / 4 \sigma)^{2}-\sigma=$ 866.25. Then, we can see that it takes short time for the system to be stabilized at origin point and the state trajectories are illustrated in Figures 2 and 3.

According to Theorem 4, we may choose $\tilde{\gamma}=100>\gamma=$ 55. Then, we can see that it takes short time for the system to be stabilized at origin point and the state trajectories are illustrated in Figure 4.

According to Theorem 5, we may choose $\widetilde{\sigma}=7>\sigma=$ 4. Then, we can see that it takes short time for the system 


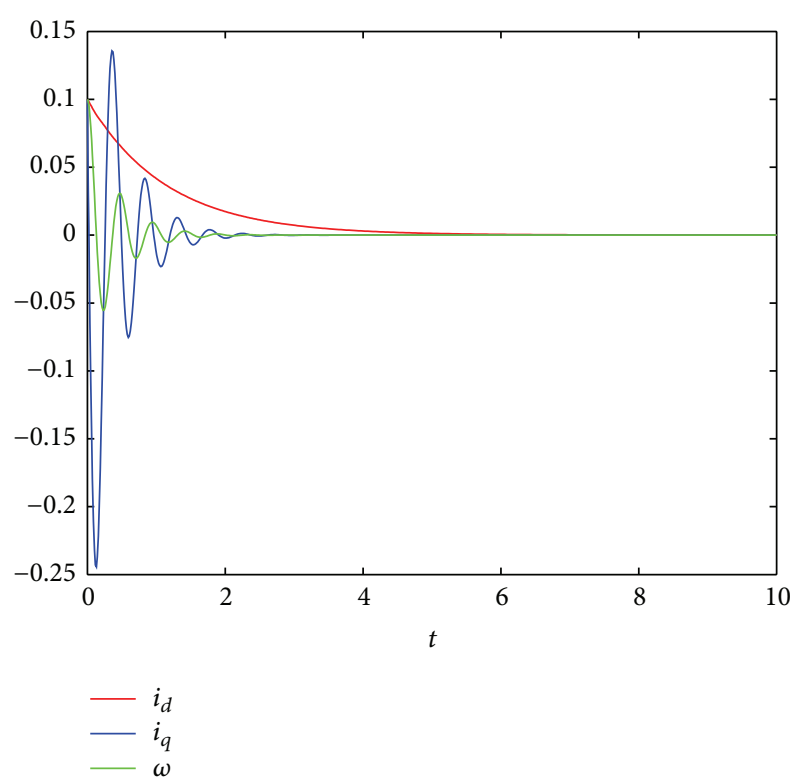

FIGURE 4: The state trajectory of $i_{d}, i_{q}$, and $\omega$ with the controller $u_{3}$.

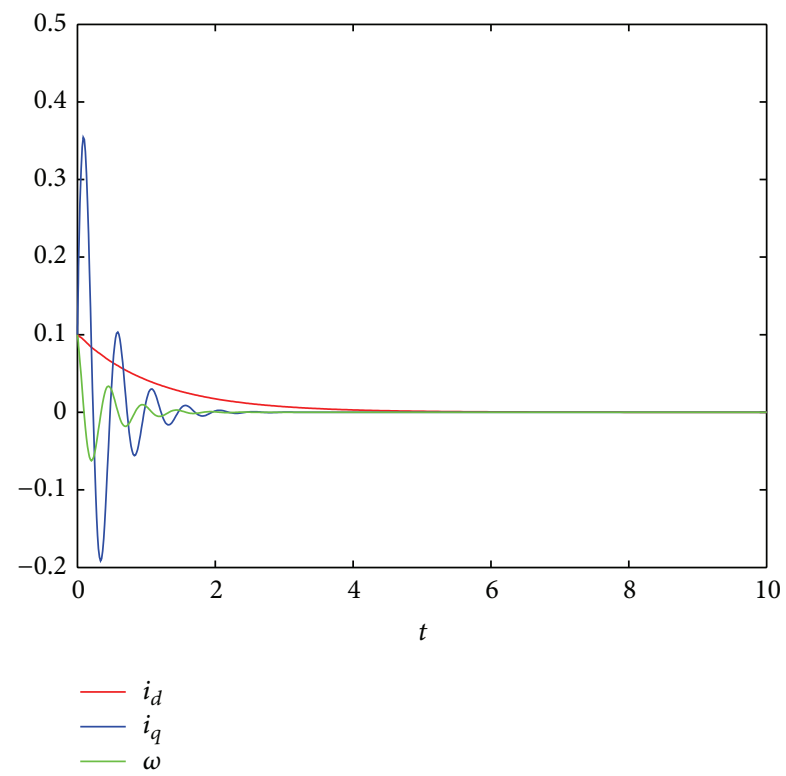

Figure 5: The state trajectory of $i_{d}, i_{q}$, and $\omega$ with the controller $u_{4}$.

to be stabilized at origin point and the state trajectories are illustrated in Figure 5.

According to Theorem 6, we may choose $\widetilde{\gamma}=54.6<\gamma=$ $55, \widetilde{\sigma}=3.2<\sigma=4$, so $0<\gamma-\widetilde{\gamma}=0.4<1,0<\sigma-\widetilde{\sigma}=$ $0.8<1$. Then, we can see that it takes short time for the system to be stabilized at origin point and the state trajectories are illustrated in Figure 6.

\section{Conclusion}

In this paper, we investigated the global exponential stabilization for BLDCM system and designed four linear and simple

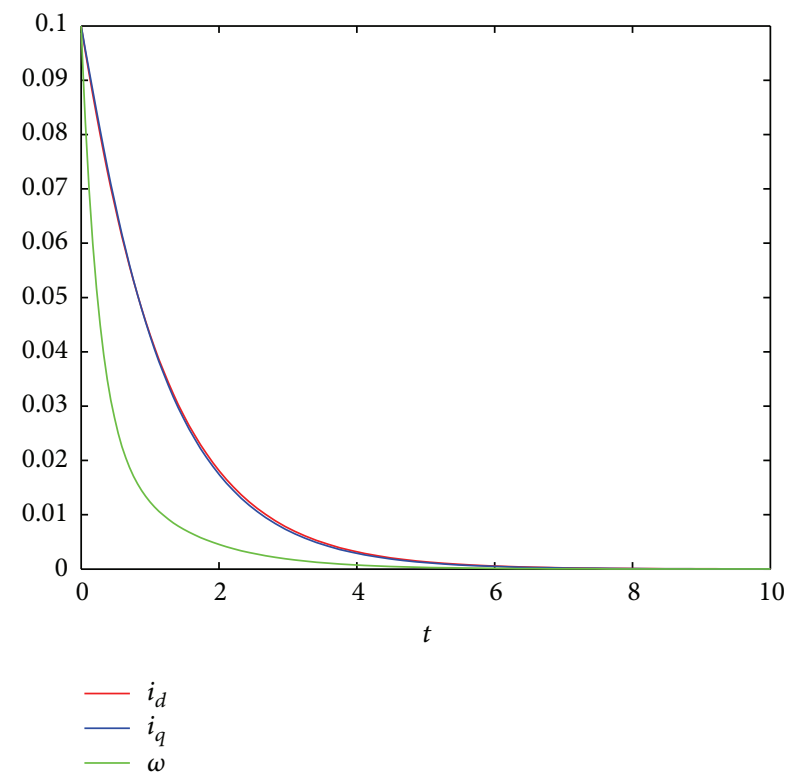

FIGURE 6: The state trajectory of $i_{d}, i_{q}$, and $\omega$ with the controllers $u_{3}$ and $u_{4}$.

feedback controllers. These controllers realized the global stabilization of BLDCM with exponential convergence rate, and the control law used in each theorem is less conservative and more concise. Finally an example is given to demonstrate the correctness of the proposed results.

\section{Conflict of Interests}

The authors declare that there is no conflict of interests regarding the publication of this paper.

\section{Acknowledgments}

This work was supported by National Natural Science Foundation of China (61340042), Natural Science Foundation of Hubei Province (2013CFC011), the Project of the Education Department of Hubei Province (T200910, T201009, and XD2014287), and the Project of the Hubei University of Science and Technology (KY13058).

\section{References}

[1] S. Dadras, F. Motallebzadeh, S. Ozgoli, and H. R. Momeni, "Control of chaotic uncertain brushless DC motors," in Proceedings of the IEEE International Conference on Control and Automation (ICCA '09), pp. 2143-2148, December 2009.

[2] Z.-M. Ge and C.-M. Chang, "Chaos synchronization and parameters identification of single time scale brushless DC motors," Chaos, Solitons and Fractals, vol. 20, no. 4, pp. 883-903, 2004.

[3] N. Hemati and M. C. Leu, "A complete model characterization of brushless DC motors," IEEE Transactions on Industry Applications, vol. 28, no. 1, pp. 172-180, 1992.

[4] N. Hemati, "Strange attractors in brushless DC motors," IEEE Transactions on Circuits and Systems I: Fundamental Theory and Applications, vol. 41, no. 1, pp. 40-45, 1994. 
[5] K. T. Chau, J. H. Chen, and C. C. Chan, "Dynamic bifurcation in DC drives," in Proceedings of the 28th Annual IEEE Power Electronics Specialists Conference (PESC '97), pp. 1330-1336, June 1997.

[6] J. H. Chen, K. T. Chau, and C. C. Chan, "Analysis of chaos in current-mode-controlled dc drive systems," IEEE Transactions on Industrial Electronics, vol. 47, no. 1, pp. 67-76, 2000.

[7] Z.-M. Ge, C.-M. Chang, and Y.-S. Chen, "Anti-control of chaos of single time scale brushless dc motors and chaos synchronization of different order systems," Chaos, Solitons \& Fractals, vol. 27, no. 5, pp. 1298-1315, 2006.

[8] G. P. Zhou, J. H. Huang, X. X. Liao, and S. J. Cheng, "Stability analysis and control of a new smooth chua's system," Abstract and Applied Analysis, vol. 2013, Article ID 620286, 10 pages, 2013.

[9] H. P. Ren and G. R. Chen, "Control chaos in brushless DC motor via piecewise quadratic state feedback," in Advances in Intelligent Computing, vol. 3645 of Lecture Notes in Computer Science, pp. 149-158, Springer, Berlin, Germany, 2005.

[10] Z. J. Meng, C. Z. Sun, Y. J. An, H. M. Yang, and Q. Hu, "Controlling chaos for BLDC thruster motor in deepwater robot based on fuzzy control," in Proceedings of the IEEE International Conference on Control and Automation (ICCA '07), pp. 712-715, Guangzhou, China, May-June 2007.

[11] B.-Q. Dong and Z.-M. Chen, "Asymptotic stability of nonNewtonian flows with large perturbation in $\mathbf{R}^{2}$," Applied Mathematics and Computation, vol. 173, no. 1, pp. 243-250, 2006.

[12] B.-Q. Dong and Z.-M. Chen, "Asymptotic stability of the critical and super-critical dissipative quasi-geostrophic equation," Nonlinearity, vol. 19, no. 12, pp. 2919-2928, 2006.

[13] Y. Uyaroğlu and B. Cevher, "Chaos control of single timescale brushless DC motor with sliding mode control method," Turkish Journal of Electrical Engineering and Computer Science, vol. 21, no. 3, pp. 649-655, 2013.

[14] D. Q. Wei, L. Wan, X. S. Luo, S. Y. Zeng, and B. Zhang, "Global exponential stabilization for chaotic brushless DC motors with a single input," Nonlinear Dynamics, vol. 77, no. 1-2, pp. 209-212, 2014.

[15] S. Kalir, S. Mangan, and U. Alon, "A coherent feed-forward loop with a sum input function prolongs flagella expression in Escherichia coli," Molecular Systems Biology, vol. 1, article 2005.0006, 2005.

[16] X. X. Liao, Theory Methods and Application of Stability, Huazhong University of Science \& Technology Press Co. Ltd., 2nd edition, 2010.

[17] Y. Q. Jia, X. G. Gui, and B.-Q. Dong, "Stability analysis of the supercritical surface quasi-geostrophic equation," Abstract and Applied Analysis, vol. 2013, Article ID 620320, 9 pages, 2013.

[18] X. X. Liao and P. Yu, Absolute Stability of Nonlinear Cntrol Systems, Springer, 2008. 


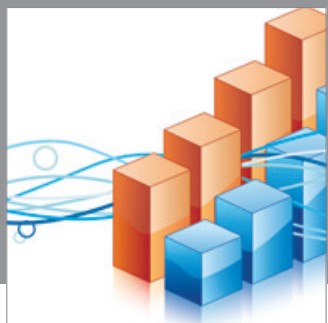

Advances in

Operations Research

mansans

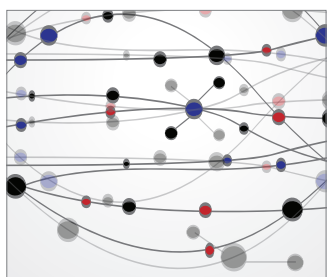

The Scientific World Journal
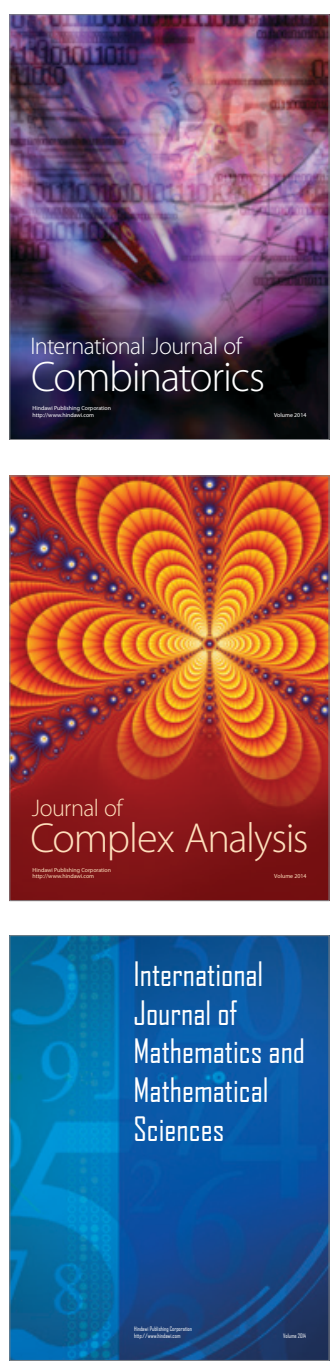
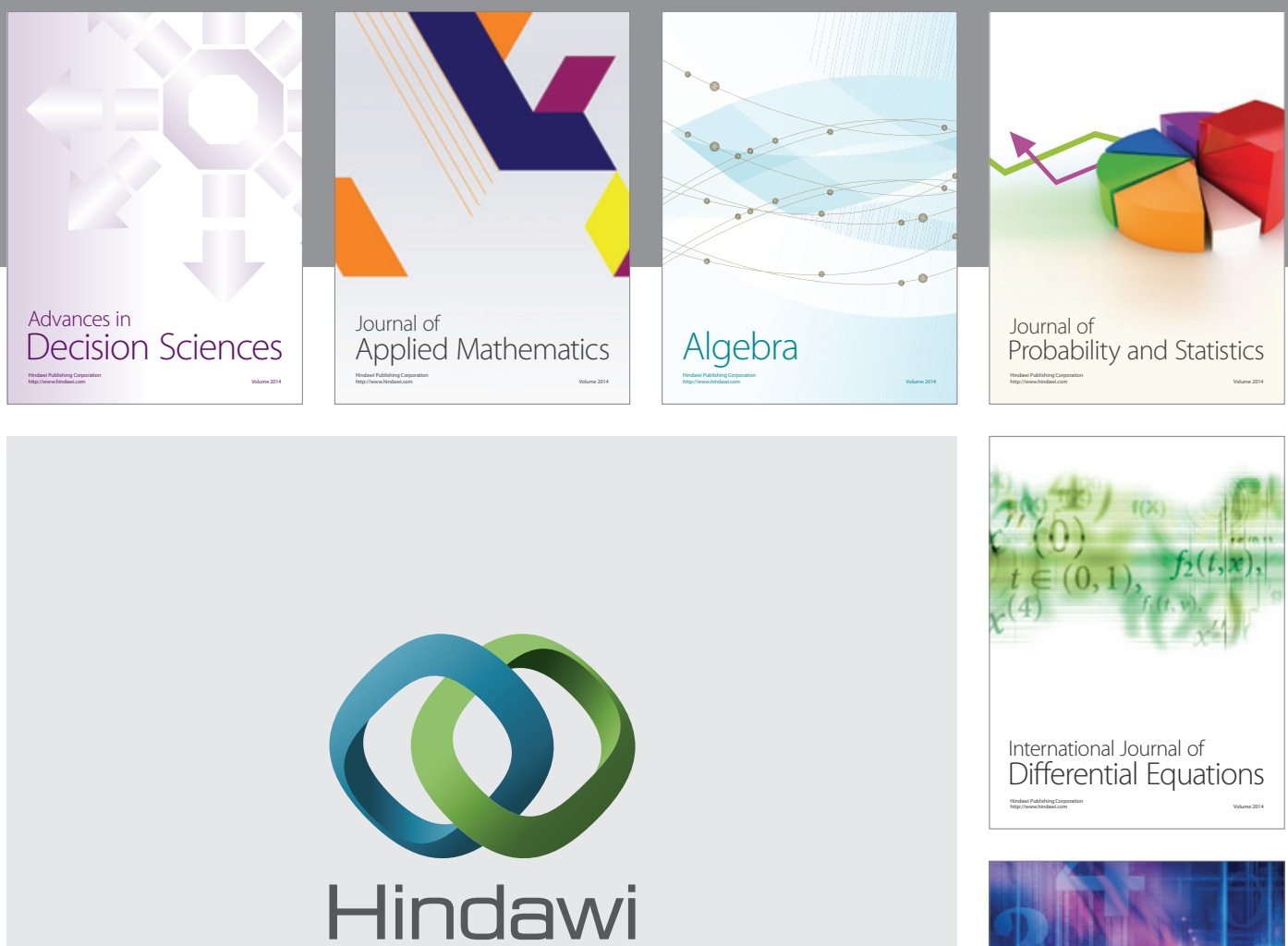

Submit your manuscripts at http://www.hindawi.com
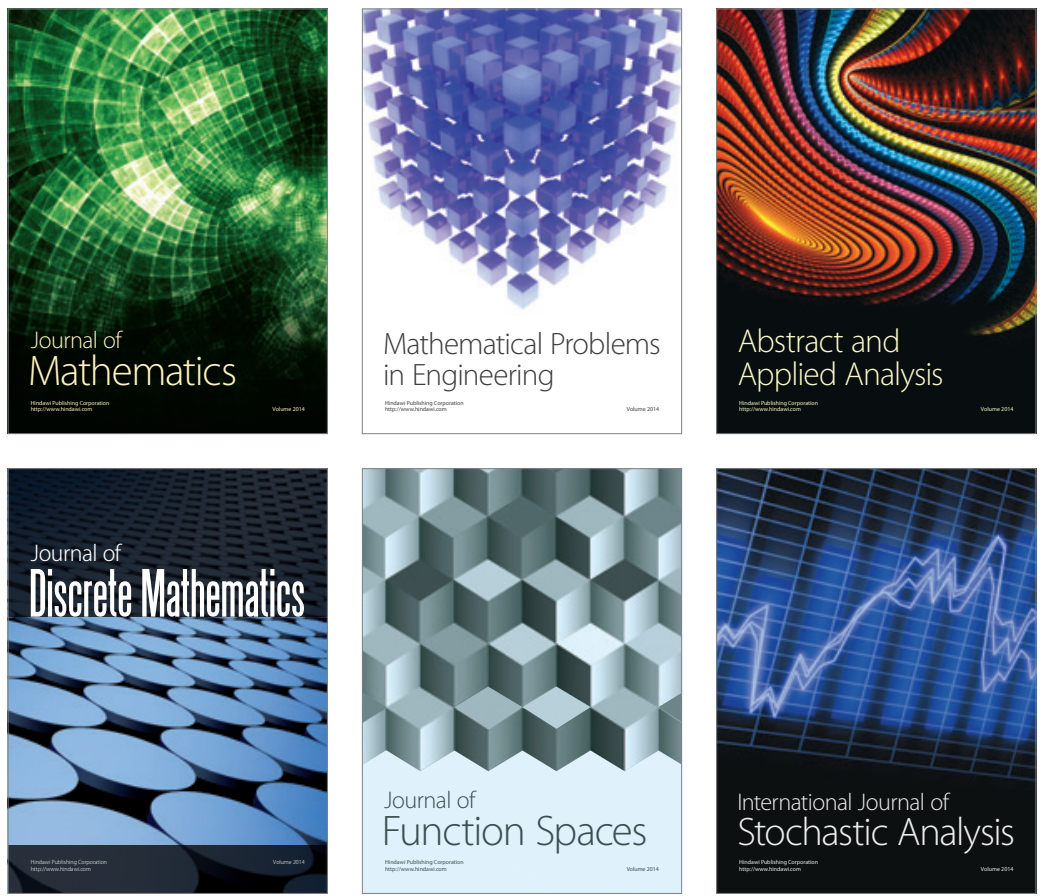

Journal of

Function Spaces

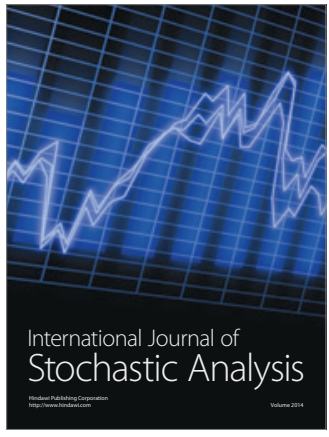

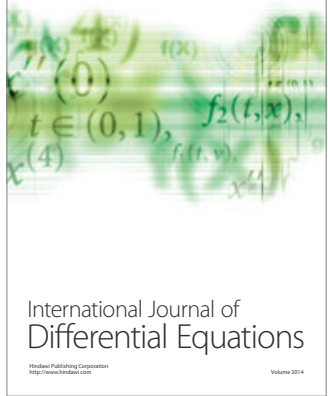
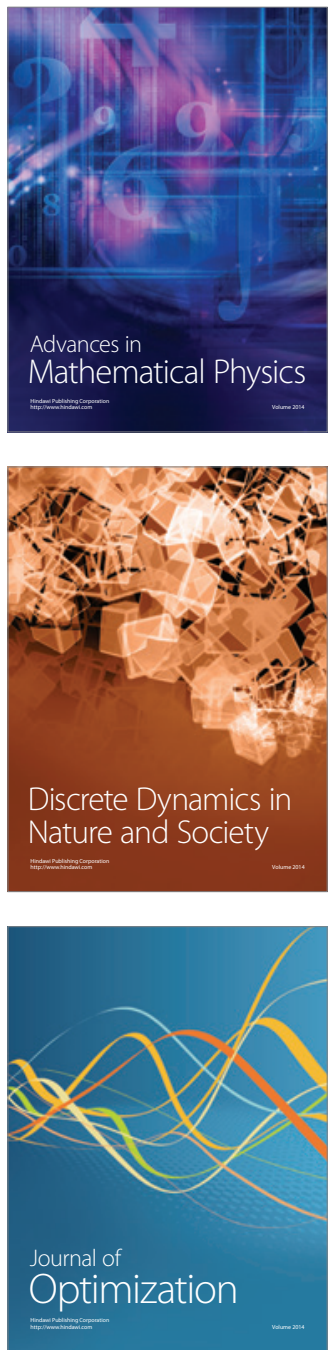